\title{
Robotic Granular Jamming: Does the Membrane Matter?
}

\author{
Allen Jiang ${ }^{1}$, Tommaso Ranzani ${ }^{2}$, Giada Gerboni², Laura Lekstutyte ${ }^{1}$, Kaspar Althoefer ${ }^{1}$, Prokar Dasgupta $^{3}$, \\ and Thrishantha Nanayakkara ${ }^{1}$
}

\begin{abstract}
Soft robotics for medical, endoscopic applications requires a dexterous and compliant mechanism to increase accessibility and decrease patient injury. However, soft structures do not offer the level of image and platform stability provided by rigid structures. Thus, a variable stiffness mechanism is an ideal solution to reconcile the two requirements of compliance and stability; the mechanism explored here is granular jamming. Granular jamming is a phenomenon where particulate matter within a membrane can transition from a fluid-like to a solid-like state, based on the level of applied vacuum pressure. In the solid-like jammed state, the conventional assumption is made that granule-granule contacts dominantly contribute to the system's stiffness. Thus, many works have evaluated the effects of different granule types by experimentally varying the sizes, shapes, and material properties of the particles. However, the role of the membrane in determining the possible range of stiffness or the variability of granular jamming has not been well studied. This paper investigates the effects and significance of membranes for a granular jamming system. Several membranes were experimentally tested and analyzed in order to find the amount of flexibility and stiffness they provide when the system is in an unjammed and jammed state, respectively. This paper presents for the first time that the membrane plays a significant contributing factor in granular jamming stiffness.
\end{abstract}

\section{Introduction}

From minimally invasive surgery (MIS) to natural orifice transluminal endoscopic surgery (NOTES), robotics has been increasingly popular in the medical field. Many hospitals are already benefiting from robot assisted laparoscopy with devices such as the da Vinci; and, for years, flexible endoscopes have aided medical practitioners to examine internal organs with minimal invasiveness. These robotic techniques have been shown to significantly improve patient recovery periods while decreasing their medical costs (1) (2). Despite these benefits, however, there are a number of hurdles that need to be addressed. For rigid arm devices, such as the da Vinci robot and traditional laparoscopic tools, instruments can clash with each other, causing additional difficulties in NOTES and laparo-endoscopic single-site surgery (LESS) (3). Flexible endoscopes, on the other hand, are preferred in NOTES and LESS, because they provide more maneuverability and have fewer trocar port requirements. However, flexible endoscopes lack the platform stability rigid devices provide (4) (5).

A variable stiffness robot takes advantage of the best properties of rigid and soft robotics. Not only will the variable stiffness robot have the stability, strength, and manipulation performance of a rigid system, but also the maneuverability and access benefits of a soft system (6). Most of the current variable stiffness robots are tendon based, and generally have issues with backlash and large external footprints (7) (8) (9) (10). This type of technology can tune their stiffness by tensioning all of its cables, but the drawback is that the tip cannot be stiffer than its base. Another popular technique stems from the control of the torque output of embedded motors. However, for a MIS application, the micromotors used do not exhibit sufficient torque for tissue manipulation (11) (12). There are several other novel techniques for achieving variable stiffness in robotics, such as the thermally activated joint (13) and pre-curved concentric tubes (14) (15). Though still new, the thermally activated joint can only achieve binary stiffness--fully soft or fully rigid--and the concentric tubes cannot dynamically adapt its stiffnesses without compromising shape.

\footnotetext{
${ }^{1}$ Centre for Robotics Research, King's College London, London, UK

2 Institute of BioRobotics, Scuola Superiore Sant'Anna, Pisa, Italy

${ }^{3}$ Department of Urology, King's College London, London, UK
} 


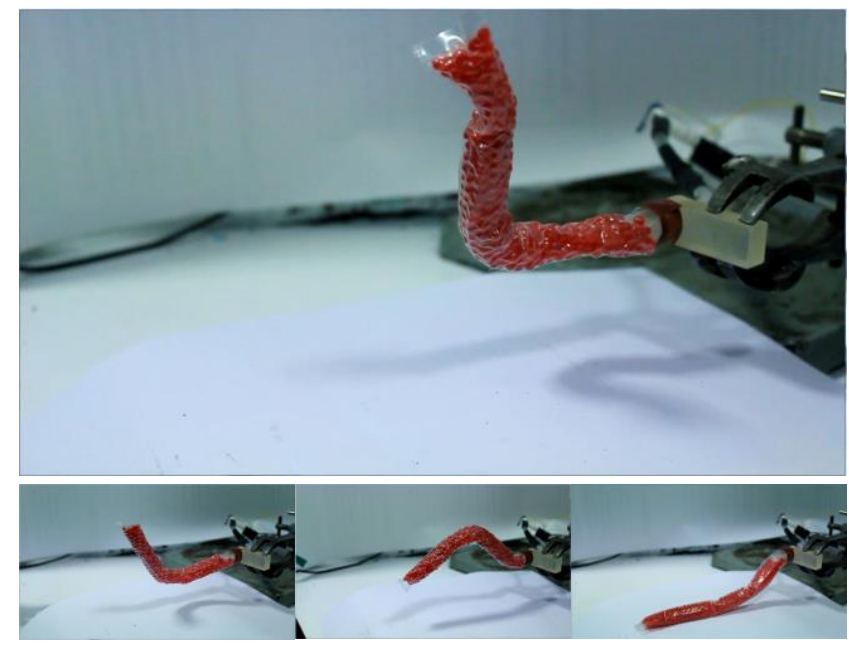

Figure 1: Prototype of a variable stiffness endoscope stiffened in arbitrary shapes (top, bottom left, bottom center) and in an unjammed state (bottom right). $4 \mathrm{~mm}$ spherical granules were used with a polythene membrane.

Also known as vacuumatics and particle mechanical constraints, granular jamming is a growing field in robotics. It is a mechanism which enables particles to act like a liquid, solid, or something in between. As stated by (16), jamming is a phenomenon where an external stress can change 'fragile matter' from a fluid to a solid-like state. A typical granular jamming mechanism consists of three parts: the particles, boundary layer membrane, and a vacuum pump. Normally, the particles are free to flow within the membrane, but when vacuum pressure is applied, the membrane "locks" the particles in place. Because of this unique feature, many groups have integrated granular jamming into robotic projects such as the universal robotic gripper (17), the tendon-supported elephant trunk (18), the jamming skin enabled locomotion robot (19) (20), the variable stiffness haptic device (21), the variable stiffness endoscope (5) (22), soft surgical manipulator (23) (24), and the emergency vacuum splint (25). Simulations have also been done by several other groups, most notably (26) (27) (28) (29) and (30). However, while these groups have examined the effects of granule size, shape, and material on the achievable stiffness range (31), the effects of the membrane are not well studied.

This paper aims to comprehensively examine several types of membrane materials suitable for a granular jamming-based medical robot. The sterilizable membranes should be soft and flexible to maintain good contact with the granules, as previous works show that such an interaction improves the hysteresis (32). Their effects on the stiffness range, hysteresis, and variability will open a broader range of granular jamming analysis, aiding in the optimization of such systems using the technology. The experimental results presented in this paper will be a first for variable stiffness robotics, and will be an aid in designs of new devices such as the Core-Snake from (33).

\section{Methods}

For the flexible endoscope, a single joint segment was analyzed to better understand the effects of the membrane during granular jamming. The joint was a cylinder $15 \mathrm{~mm}$ in diameter and $40 \mathrm{~mm}$ in length, filled with $4 \mathrm{~mm}$ diameter glass spheres. The average packing factor for each test was $0.61+/-0.01$. As the paper is focused on the membranes, a reference material of glass was chosen for the granules. Glass was used for its elastic and frictional properties, as well as being a classic granule type in literature. The $4 \mathrm{~mm}$ diameter size was chosen based on the author's previous work, which showed that $4 \mathrm{~mm}$ is an ideal size for the given joint dimensions (32) (34).

To comprehensively record the behavior and effect of membranes in granular jamming, three types of tests were performed on the granular jammed joint: bending, tensile, and compression tests. These tests are meant to categorize the behaviors of the joint from tasks the robot will perform. Because the granules and membranes are decoupled, the joint may behave as different equivalent materials for the three types of tasks it must perform. For example, in turning or lifting, the behavior of the joint may have a different stiffness characteristic than for pulling or pushing. The bending tests 
correspond to these turning or lifting manipulation tasks, the tension tests correspond to pulling tasks, and the compression tests correspond to pushing tasks the joint and robot will perform.

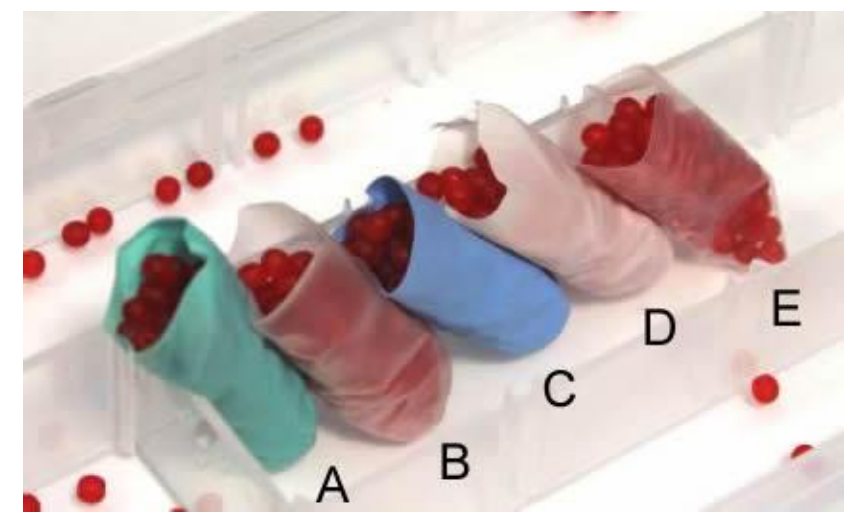

Figure 2: The various membranes tested for their effect on a granular jamming joint were, from left to right: A) vitrile, B) vinyl, C) nitrile, D) latex, and E) polythene.

The five different membranes tested were the following: latex, nitrile, vinyl, vitrile, and polythene. The former four materials were chosen based on their applications in the medical field, particularly surgical gloves. Latex is the most commonly used surgical membrane, with nitrile and vinyl commonly used as latex-free substitutes. Vitrile is a mixture of vinyl and nitrile, offering increased strength over pure vinyl and increased flexibility over pure nitrile. The polythene material was chosen from its applications in the food industry, such as vacuum packaged goods. Polythene is a lowdensity polyethylene, the most commonly used non-toxic plastic material. Figure 2 shows the five material types selected for this paper.

The experiments were repeated at three different pressure levels: $101 \mathrm{kPa}$ (15 PSI-A), $55 \mathrm{kPa}$ (7.5 PSI-A), and $10 \mathrm{kPa}(1.5$ PSI-A). The pressures were achieved by a two-stage, oil-based vacuum pump (Mastercool 90066-2V-220) and measured by an absolute pressure sensor (Honeywell 0-30 PSI). Thus, all pressure measurements were done in absolute pressure.

Additionally, tensile tests were performed on the membranes to quantify the Young's modulus of each material. These experiments were done at atmospheric pressure (101 kPa/15 PSI-A) without granules.

For the bending tests, the joint tip was deflected horizontally by a motorized linear rail for a distance of $10 \mathrm{~mm}$ at a speed of $1 \mathrm{~mm} / \mathrm{sec}$, as seen in Figure 3. The resistive force done by the joint was measured by an ATI Nano17 Force/Torque Sensor. This experimental setup was controlled and recorded with LabVIEW. Ten experimental trials were performed for each membrane at each pressure level.

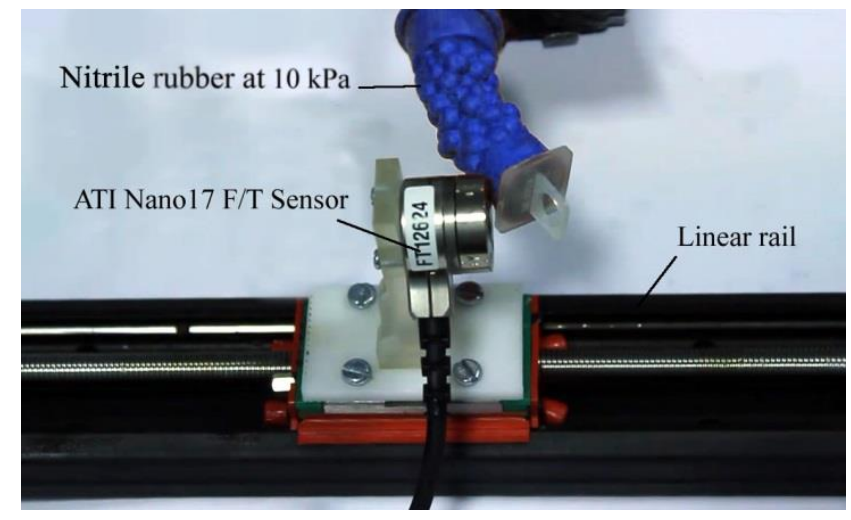

Figure 3: The bending experimental setup, where the joint tip is deflected $10 \mathrm{~mm}$ horizontally. 
The tensile experimental setup can be seen in Figure 4. The ends of the joint are held in place by grippers, and are deflected with a $20 \%$ strain. The velocity of the tensioning was $10 \mathrm{~mm} / \mathrm{min}$. Five experimental trials were performed for each membrane at each pressure level.
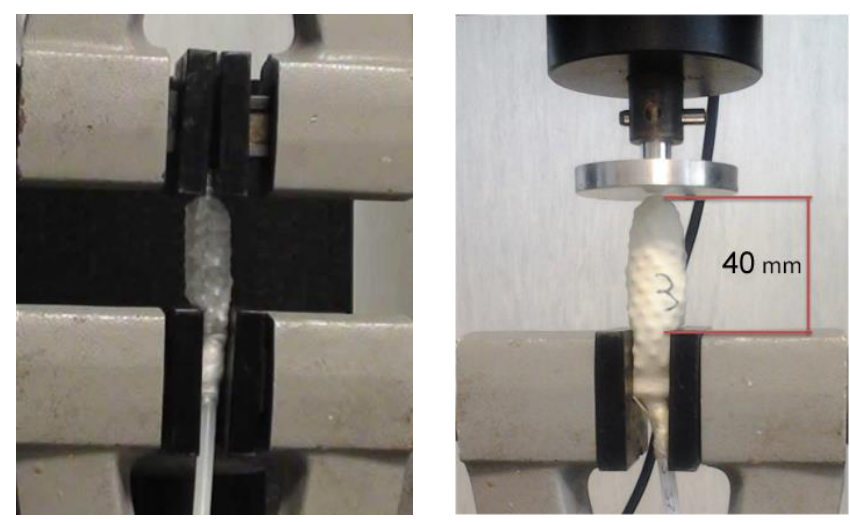

Figure 4: Experimental setup for the tensile and compressive tests, where the joint is deflected with a $20 \%$ strain in tension and compression, respectively.

Figure 4 also shows the compression experimental setup. Similar to the tensile experiments, the joint's base was fixed using grippers, and a displacement of $20 \%$ strain was performed. The velocity of the compression tests were also set to 10 $\mathrm{mm} / \mathrm{min}$. Five trials were performed on each membrane for each of the three pressure levels.

The tension and compression experiments were done with an Instron 5900 Testing System. Data from the first trial of each experiment with the Instron were not used, due to the Mullins effect (35).

The hysteresis value $\mathrm{H}$ is normalized for each experiment and is calculated by the difference in area of the loading (top) and unloading (bottom) curves divided by the area of the loading curve $\left(H=A_{\text {load }}-A_{\text {unload }} / A_{\text {load }}\right)$.

\section{Results}

The individual results show the measured force or stress for given deflections, the hysteresis, and the variability for each of the membranes under each test to underline the differences between membrane materials. Additional analyses were performed to summarize the relationship between pressure and effective Young's modulus for each membrane type from the experimental results for the bending, tensile, and compression tests, respectively.

In the results, the value of the Young's modulus $\mathrm{E}$ is used to derive common interpretations the stiffness property of the joint from three types of tests: bending, tension, and compression. Because of the unique structure of the granular jamming joint, the modulus $\mathrm{E}$ differs among the different deformation types. This gives rise to design guidelines for membrane material selection depending on the importance of each type of deformation a robot would experience.

\section{Membrane tensile analysis}

To see the behavior of the membrane and empirically measure the effective Young's modulus E, tensile tests were performed on the membranes themselves. Young's modulus E was calculated by the slope of a linear plot fitted onto the experimental results, as E=stress/strain. The empty membrane cylinders were subjugated to $20 \%$ strain. The thicknesses of the membranes were $0.07 \mathrm{~mm}$, with the exception of the polythene, which was $0.06 \mathrm{~mm}$ in thickness. The volume of the membrane cylinders were all $7070 \mathrm{~mm}^{3}$. 

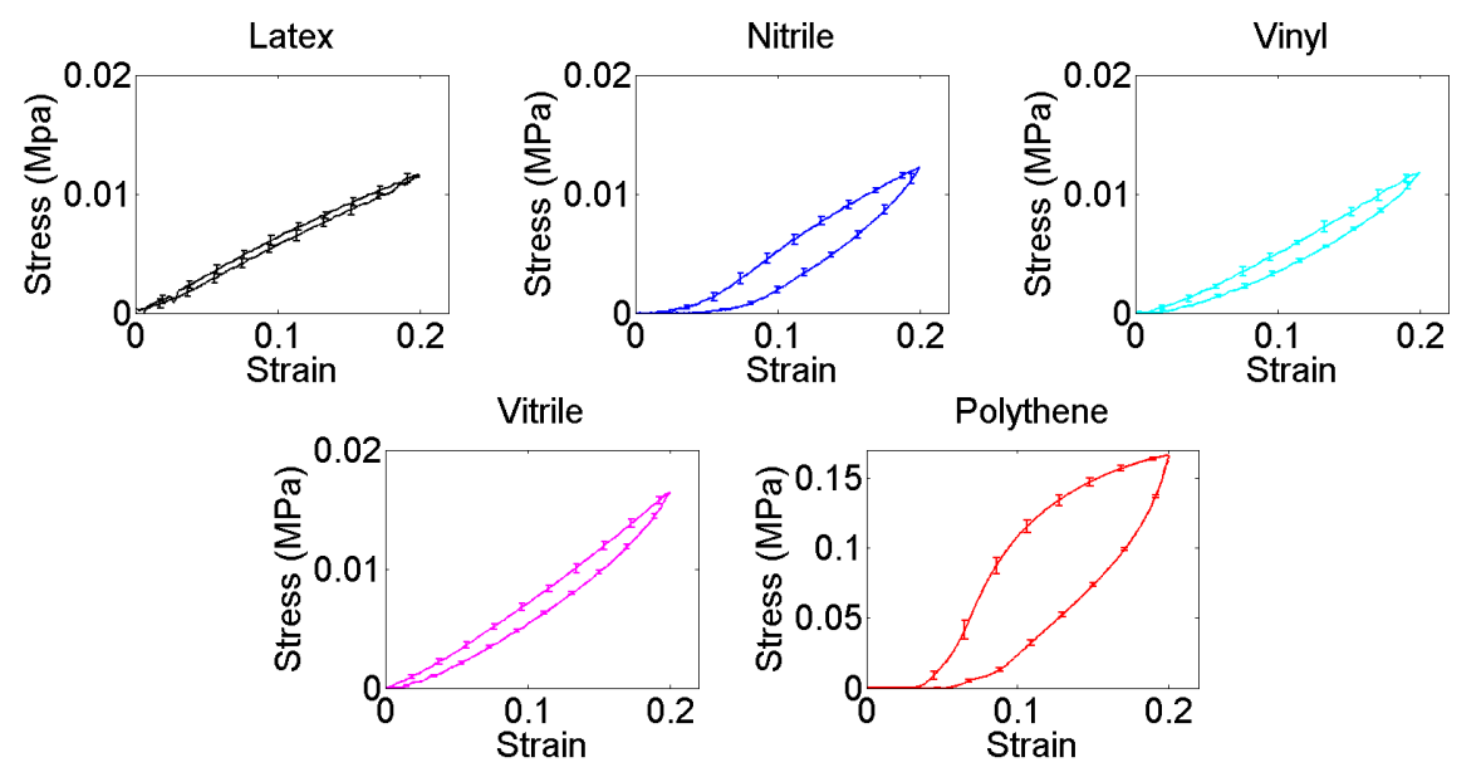

Figure 5: Five different membranes tested with $20 \%$ tensile strain. (Note: Polythene is on a different scale to show detail)

Under tensile strain, latex, vinyl, and vitrile exhibit fairly linear behavior with very little hysteresis, as shown in Figure 5 . Nitrile has a relatively large hysteresis, similar to that of the polythene. In the case of polythene, E was still derived from a fitted linear curve, despite the nonlinear behavior. This approximation was taken as it exhibited ten times greater stress than the former four membrane types and the resulting E value also showed to be similarly greater. From observation, the polythene material's large hysteresis is due to permanent deformation caused by stretching the membrane.

Performing a pairwise Mann-Whitney $U$ test on the peak stresses, the latex-vinyl and nitrile-vitrile pairs were not significantly different $(\mathrm{p}>0.2)$. All other pairs were found to be significantly different $(\mathrm{p}<0.008)$.

\section{Bending results}

In Figure 6, polythene indeed showed to achieve the highest bending force at $3.12 \mathrm{~N}$, about $30 \%$ greater than the next highest, nitrile. However, this was at a cost of hysteresis and variability. This shows that a ten-fold increase in E, as seen in Figure 5, does not increase the bending force by the same amount. The poor performance in hysteresis is due to the high level of permanent deformation the joint undergoes after being loaded, but not from the membrane itself; namely, the interaction between the granules and the membrane, as well as granules to granule interactions, caused the joint as a whole to deform permanently. This does suggest that the membrane is too stiff to possess the elasticity to reverse the motion of the internal particles, though the membrane itself does not permanently deform. At $7 \mathrm{~mm}$, the joint is no longer in contact with the force sensor on the return trip, and thus no forces are recorded. On the same macro scale, each of the joints exhibit similar amounts of hysteresis at atmospheric pressure. Without a pressure differential, the resistance to bending and the elasticity of the joint is dominated by the membrane, rather than the granules. Nitrile and latex have the second and third highest stiffness ranges, and they perform better than polythene in terms of both hysteresis and variability. Those two materials perform similarly across all the measured parameters with each other, as well. Vinyl and vitrile have lowest stiffness ranges, and have similar amounts of variability with latex and nitrile. 

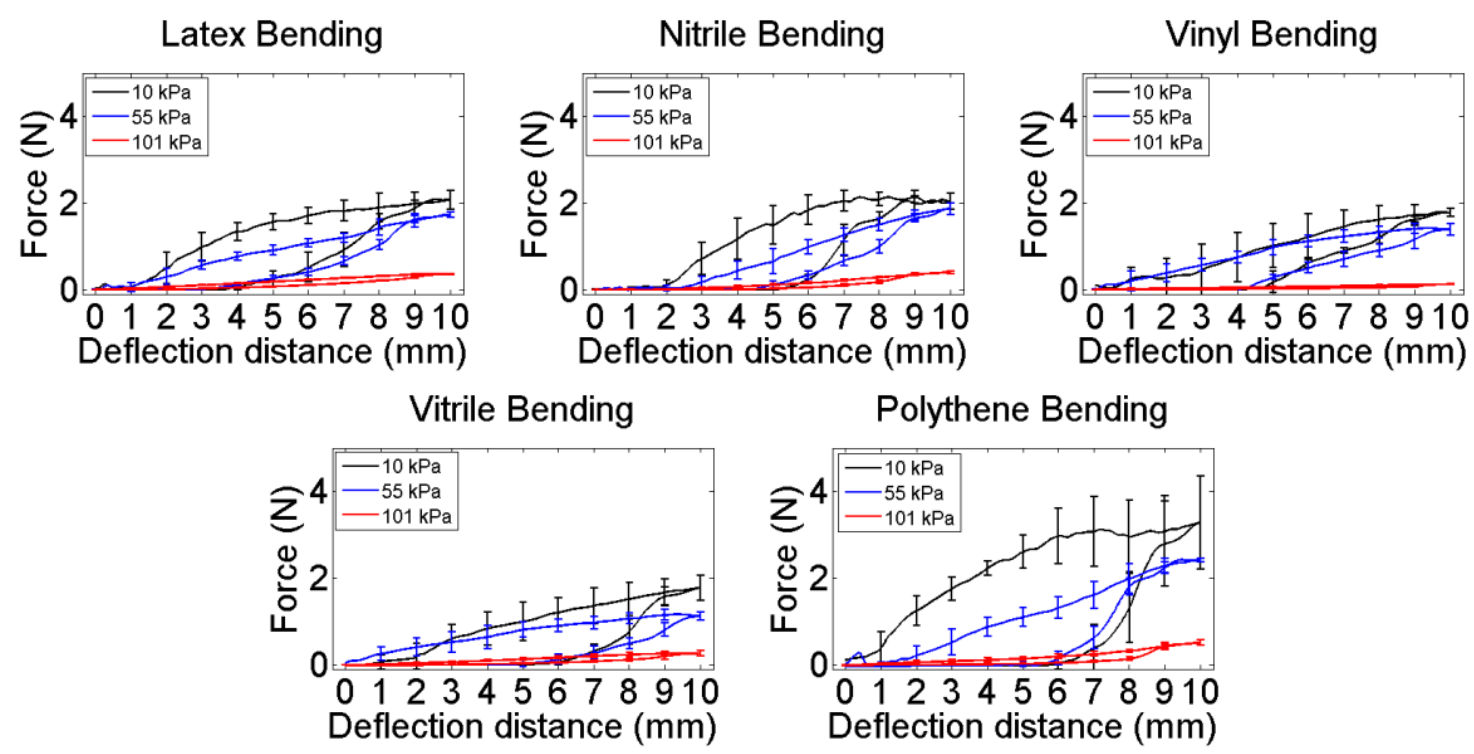

Figure 6: Bending experimental results from the five membrane types (latex, nitrile, vinyl, vitrile, and polythene) for three internal pressures $(101,55$, and $10 \mathrm{kPa})$, with $4 \mathrm{~mm}$ smooth glass spherical granules.

The variability between trials was about $10-12 \%$ of the measured force for each membrane at $10 \mathrm{kPa}$. Thus, while hysteresis may be high per trial, there is a level repeatability between trials. We performed a pairwise Mann-Whitney U test to ascertain whether the influence of different membranes on the variability of the peak stiffness of the joint were significantly different from each other. In the unjammed state (pressure $=101 \mathrm{kPa}$ ), all pairs were significantly different with the latex-vitrile pair of $p<0.005$, and all other pairs giving $p<0.0003$. Since the peak stiffness of the unjammed state mainly depends on the membrane stiffness, this implies that there is a base level difference in the bending stiffness due to the different types of membranes we selected for this experiment. Some pairwise comparisons changed their level of statistical significance when we applied the maximum level of vacuum (10 kPa), such as with vinyl and vitrile losing a significant difference ( $p$ > 0.8). This implies that there is an interaction effect between the type of membrane and the level of vacuum. In order to test whether there is a significant overall interaction effect between pressure and membrane type, we performed an n-way ANOVA test. We found that, while both the pressure and membrane type have a significant effect on the stiffness of the joint separately ( $\mathrm{p}<0.0001)$, there is also a significant interaction effect between the membrane type and pressure $(\mathrm{p}<0.0001)$.

In addition to the statistical tests on the peak lateral force, we tested whether membrane type causes a significant difference in the force/strain profiles shown in Figure 6. Each force profile was significantly different from others ( $\mathrm{p}<$ $0.0001)$ for the $10 \mathrm{kPa}$ pressure level. For the $101 \mathrm{kPa}$ pressure level, nitrile and vitrile are similar ( $\mathrm{p}>0.1)$.

To estimate the stiffness parameter of the granular jamming joint, we approximated it as a one end fixed cantilever beam undergoing a force at the tip. The purpose of these estimations is to provide a first look at the effect of Young's modulus $E$ in the context of granular jamming, despite the joints exhibiting some inelastic behavior. Note that this model does not estimate the hysteresis from the unloading phase. The total bending moment $M$ is the following:

$$
|M|=|L|\left|F_{\text {ext }}\right|
$$

where $M$ is the total moment, $L$ is the length of the beam, and $F_{\text {ext }}$ is the externally applied force. For our bending experiments and stiffness parameter estimation based on the cantilever assumption, $L$ was $40 \mathrm{~mm}$. The moment at single point along the beam is characterized by the following:

$$
M=F_{\text {ext }}(L-d)
$$


where $d$ is the distance from the fixed end. From Equation 2 we can see that the change in moment decreases linearly, as $d$ approaches the tip. Thus, with the fixed end undergoing the largest moment, the jammed system will bend the most at the base, as seen in Figure 3.

The beam bending behavior can be written as a fixed-ended solid cylinder undergoing a point load at the free tip, and it is written as follows:

$$
y(d)=\frac{F_{e x t} d^{2}(3 L-d)}{6 E I}
$$

where $y(d)$ is the perpendicular displacement of the beam along distance $d$. Figure 7 shows the calculated beam bending shape and corresponding E value from Equation 3, where the tip deflection distance $y(L)$ is kept constant at 10 $\mathrm{mm}$ and $F_{\text {ext }}$ is from experimental data.
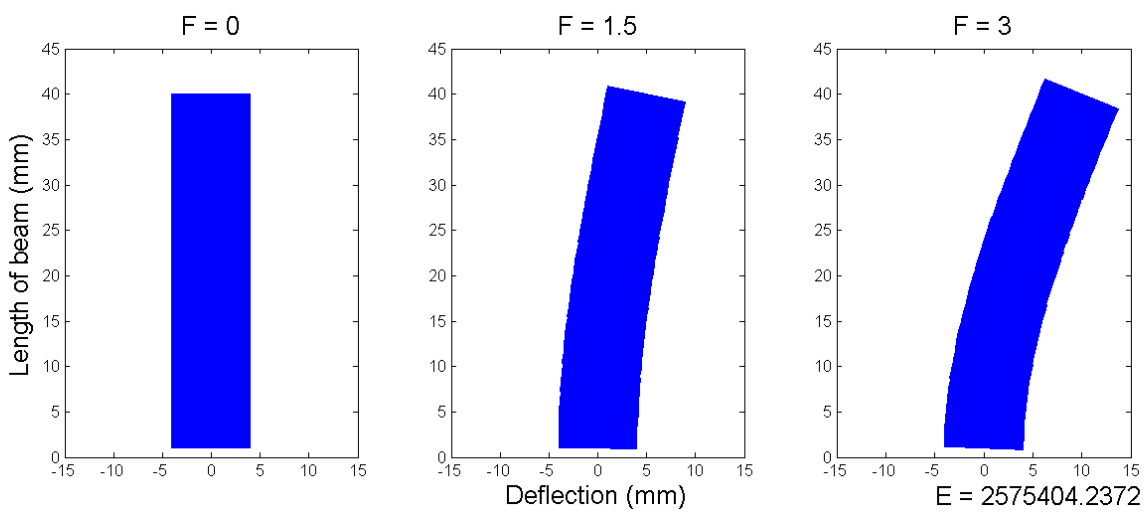

Figure 7: Calculated beam bending to find the equivalent Young's modulus E, given a tip force $\boldsymbol{F}_{\text {ext }}$ and tip displacement $y(L)$

The area moment of inertia $I$ is given as:

$$
I=\frac{\pi r^{4}}{4}
$$

with $r$ as the radius of the joint.

Thus, from the experimental bending results, and rearranging Equation 3, E can be calculated as:

$$
E=\frac{4 F_{e x t} L^{3}}{3 \pi y(L) r^{4}}
$$

Figure 8 summarizes the $\mathrm{E}$ values for each of the membranes under different pressures. Latex and nitrile exhibited similar properties, while vinyl and vitrile also behaved similarly. Polythene showed the largest E, indicating that the membrane's E value correlates to the elasticity and stiffness of the joint as a whole. 


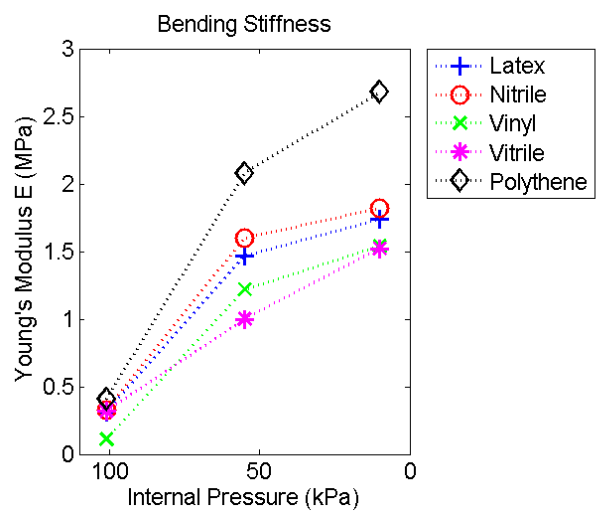

Figure 8: Relationship between vacuum pressure and stiffness for bending

\section{Tensile results}

The tensile tests demonstrate that, in tension, the mechanical properties of the membrane affect the performance of the joints in jammed states. However, as the granules do not exhibit cohesion, the question remains, does jamming have an effect on the joint's stiffness? At an atmospheric $101 \mathrm{kPa}$ internal pressure, the joint behaved similarly to the membraneonly tensile test. However, vacuum pressures altered the joint's stiffness characteristics.

Figure 9 show that the mechanical properties of latex, nitrile, and vinyl are rather similar, but nitrile presents a larger hysteresis. On the other hand the polythene is one order of magnitude stiffer than the other membranes and for a $20 \%$ strain, it undergoes permanent deformation thus presenting a higher hysteresis. In general, the granule-filled joints in these tensile tests present an elastic (linear) behavior for small deformation, after which the slope of the graphs changes considerably. Indeed, the joint tends to act as a unique material; after this linear region the mechanical behavior of the joint can be compared to a sample after reaching its yield stress. The internal particles have been separated from the applied load. According to this interpretation, the joint in stiffness varying applications should be used in the pseudoelastic tract in order to take advantage at best of the different performances. Thus, the joint should be operated for a range of deformation that is different at each vacuum level.
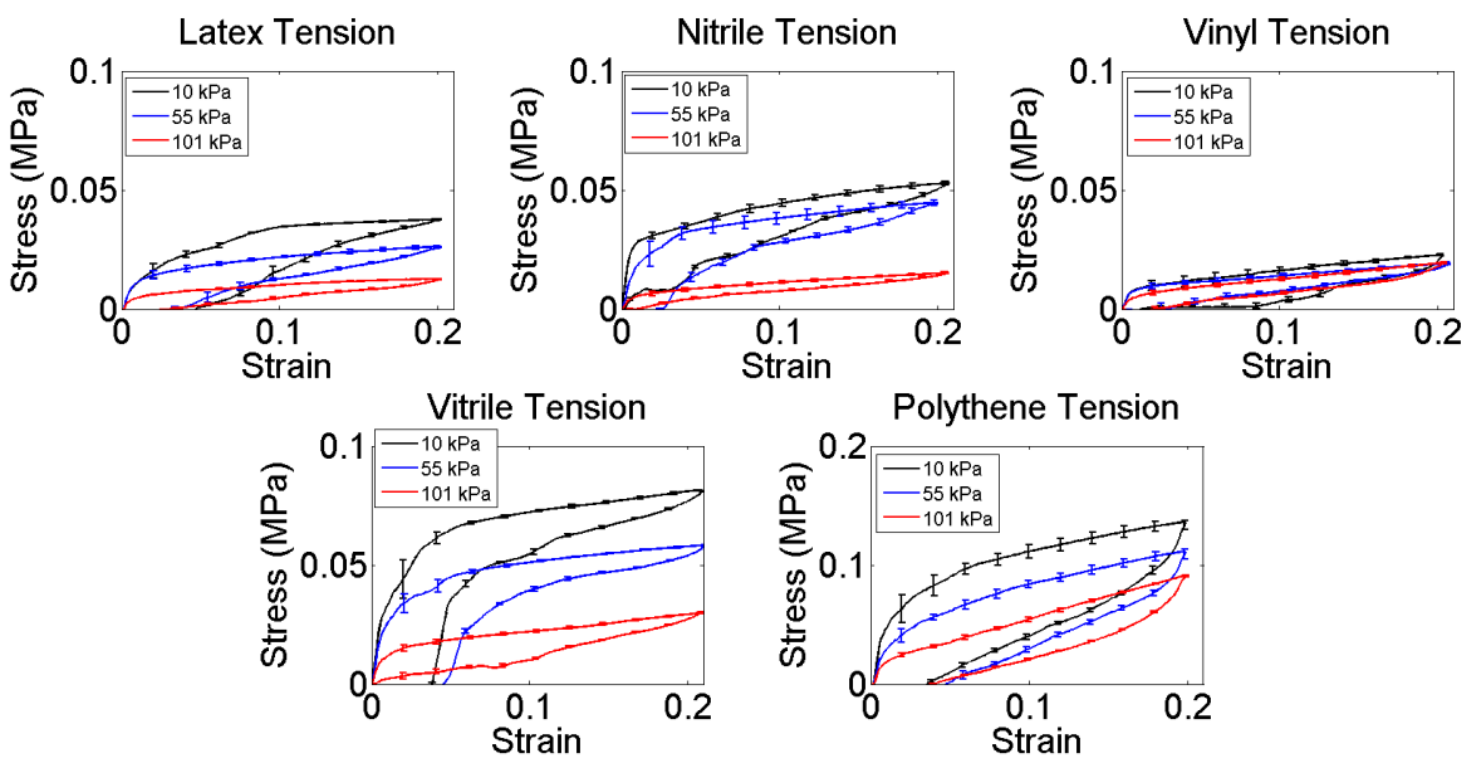

Figure 9: Tensile experimental results from the five membrane types (latex, nitrile, vinyl, vitrile, and polythene) for three internal pressures (101, 55 , and $10 \mathrm{kPa}$ ), with $4 \mathrm{~mm}$ smooth glass spherical granules. (Note: polythene is on a different scale for detail)

The latex membrane filled with glass spheres in the case of atmospheric pressure presents a trend similar to the membrane alone. When $55 \mathrm{kPa}$ vacuum is applied the slope of the initial elastic tract more than doubles from 0.56 MPa to $1.6 \mathrm{MPa}$; when the vacuum is increased to $10 \mathrm{kPa}$ the slope of the initial tract further increase to $1.8 \mathrm{MPa}$. Hysteresis in the case of 
the test performed at atmospheric pressure is 0.0038 and increases considerably when vacuum is applied to 0.0108 at 55 $\mathrm{kPa}$ up to 0.0138 at $10 \mathrm{kPa}$, confirming that a high level of energy is dissipated in the jammed configuration due to internal friction and permanent deformation of the membrane.

The elastic properties of latex and nitrile behaved similarly at atmospheric pressure. When vacuum is applied, the nitrile stress-strain slopes of the elastic tract increase to $1.8,3$, and $6.3 \mathrm{MPa}$ for the pressure levels 101,55 and $10 \mathrm{kPa}$, respectively. It is interesting to observe that, at the $10 \mathrm{kPa}$ pressure level, the nitrile elastic tract extends to a higher deformation than in the case of latex.

When at atmospheric pressure, the Young's modulus E of the joint with each membrane are similar to the values found in the membrane-only tests, apart from polythene. Thus, without jamming, the tensile strength of the joint is limited by the membrane, with granules playing little effect. However, with vacuum, latex, nitrile, and vitrile doubled and tripled their $\mathrm{E}$ values. Vinyl displayed no significant change, and polythene increased by $50 \%$.

From the variability standpoint, the variability between trials was fairly low, 1-2 orders of magnitude smaller than those from the bending tests.

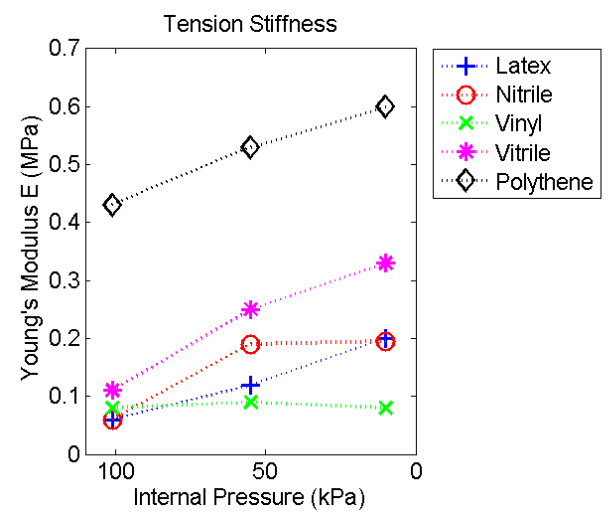

Figure 10: Relationship between vacuum pressure and stiffness for tension

Figure 10 shows some materials, such as nitrile, vinyl, and vitrile decrease in stiffness from 55 to $10 \mathrm{kPa}$. On the other hand, latex and polythene increase linearly as more vacuum is drawn.

Performing pairwise Mann-Whitney $U$ tests on the peak stresses show that, at $101 \mathrm{kPa}$, all membranes were significantly different from each other $(p<0.008)$, with the exception of nitrile and vinyl ( $p>0.4)$. At $10 \mathrm{kPa}$, these membranes, like all other pairs, become significantly different $(\mathrm{p}<0.008)$. This indicates that though membranes may exhibit similar properties when the joint is unjammed, they become distinct when vacuum is applied implying a significant interaction effect between the membrane types and level of vacuum.

The Mann-Whitney U tests for the stress/strain profiles show that, at $10 \mathrm{kPa}$, each membrane is significantly different from the others $(\mathrm{p}<0.0001)$, apart from latex and nitrile $(\mathrm{p}>0.1)$. At $101 \mathrm{kPa}$, latex is similar to nitrile and vinyl $(\mathrm{p}>0.2)$, and nitrile is somewhat similar to vinyl ( $\mathrm{p}>0.05)$.

\section{Compression results}

In most elastic materials, such as rubber, Young's modulus can be found from tension or compression. However, because the structure of the joint is composed of granular material, it is possible for the joint to behave differently under compression than under tension.

The compression tests differ largely from either the bending or tensile tests, as the joints show relatively little stress until 0.15 to 0.18 compressive strain. The reason for this is likely two-fold. The first is due to the granules having restructured and repacked to a different jammed state after the first cycle. The dotted lines in Figure 11 show this occurring, as granule rearrangement can be seen in drops in stress. Secondly, the membrane could have undergone permanent changes, known 
as the Mullins effect, after the first cycle. As the membrane behavior stabilizes after this first cycle, the analysis is done on the subsequent trials.

In Figure 11, the hysteresis of each membrane varies significantly between atmospheric and vacuumed pressures. In atmospheric conditions, the values of hysteresis for all the membranes are about 2-3 times larger than at medium vacuum. Vitrile and polythene have similar hysteresis values at $10 \mathrm{kPa}$ with atmospheric pressure, whereas the other membranes do not show such an increase in hysteresis. Through observation, the higher hysteresis at atmospheric pressure can be attributed to the membranes' inability to return to its initial shape when the Instron returns to its initial position. However, at $55 \mathrm{kPa}$, the stress the membrane imposes on the granules enables them to maintain the joint structure after the Instron moves back. This same behavior is observed at $10 \mathrm{kPa}$ for latex, nitrile, and vinyl. For vitrile and polythene, the $20 \%$ compressive strain is observed to cause some permanent deformation in the joint, possibly from a restructuring of the granules.

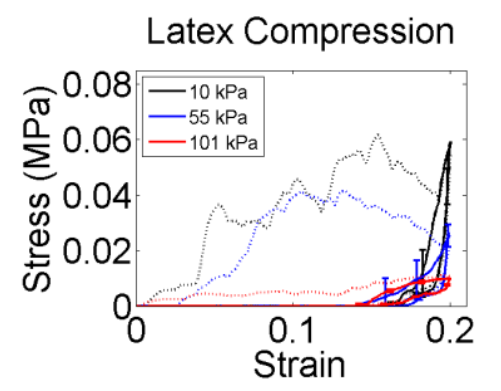

Vitrile Compression
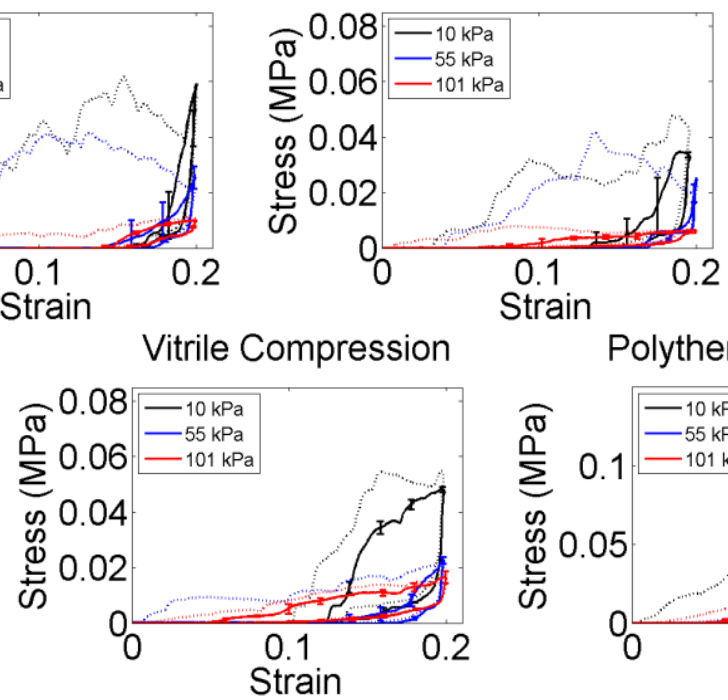

Polythene Compression

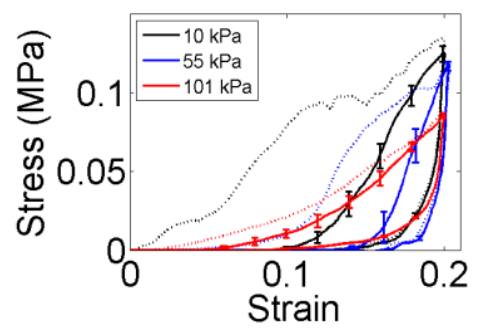

Figure 11: Compression experimental results from the five membrane types (latex, nitrile, vinyl, vitrile, and polythene) for three internal pressures $(101,55$, and $10 \mathrm{kPa})$, with $4 \mathrm{~mm}$ smooth glass spherical granules. The dotted lines represent the first loading cycle, which was omitted from the analysis due to the Mullins effect. (Note: polythene is on a different scale for detail)

Like the tensile results, from the variability standpoint, the variability between trials was fairly low, 1-2 orders of magnitude smaller than those from the bending tests.

Young's modulus E was calculated from the slope of the non-zero values between 0.18 to 0.2 strain.

Figure 12 shows that polythene was not significantly stiffer than other materials at $10 \mathrm{kPa}$, with vinyl exhibiting the same stiffness of $\mathrm{E}=3.3 \mathrm{MPa}$. In fact, vinyl showed a drastically larger stiffness range than any other material, with latex a close second. Nitrile and vitrile behaved very similarly in this test. 


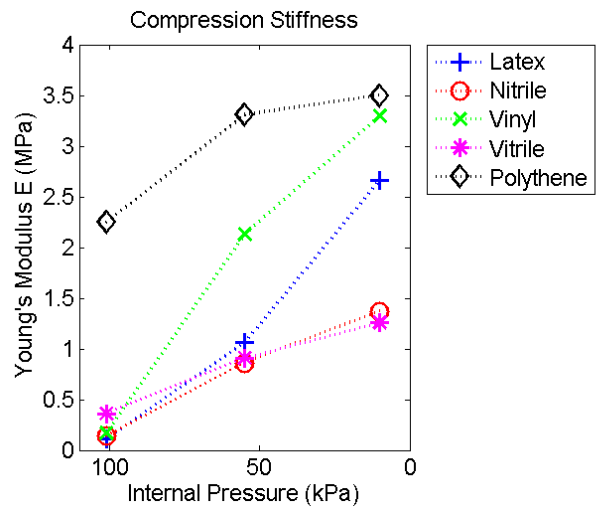

Figure 12: Relationship between vacuum pressure and stiffness for compression

The pairwise Mann-Whitney $\mathrm{U}$ tests for the peak stresses show that, at $101 \mathrm{kPa}$, each pair is significantly different ( $\mathrm{p}<$ $0.008)$, though nitrile and vinyl were only mildly different $(\mathrm{p}<0.01)$. At $55 \mathrm{kPa}$, several membrane pairs converge, with latex, nitrile, and vinyl behaving similarly ( $\mathrm{p}>0.1)$. However, at $10 \mathrm{kPa}$, all membranes become significantly different from each other $(\mathrm{p}<0.008)$, implying that at high vacuum levels, there is a significant interaction effect between the membrane types and the level of vacuum.

The Mann-Whitney U tests for stress/strain compression profiles shows that vinyl and vitrile are mildly similar $(\mathrm{p}>0.05)$ at $10 \mathrm{kPa}$, with all other membrane combinations are distinct $(\mathrm{p}<0.005)$. At $101 \mathrm{kPa}$, the five membranes were significantly different from each other $(\mathrm{p}<0.005)$.

\section{Discussion}

The comprehensive tests on latex, nitrile, vinyl, vitrile (blend of vinyl and nitrile), and polythene performed in this paper spark many interesting points in the world of robotic granular jamming. The material of the membrane affects not just the stiffness range, but the variability and hysteresis, as well. From the data analysis, several membranes were not significantly different from each other without the presence of vacuum pressure, as exhibited by the pairwise MannWhitney $\mathrm{U}$ tests on membrane-only, $101 \mathrm{kPa}$ tensile, and $101 \mathrm{kPa}$ compression tests. However, with the addition of granules and vacuum pressure, the membranes diverge to become distinct with statistically significant differences. On the other hand, in bending, the membranes were distinct at the $101 \mathrm{kPa}$ level, but had a converging pair at $10 \mathrm{kPa}$. This implies that there is a different type of interaction between the pressure, granules, and membranes in bending than in tension or compression. Thus, future designs for granular jamming manipulators must consider the principle motions it will exhibit before selecting a membrane type.

In the case of the polythene, a high stiffness was achieved, likely due to its high tensile modulus characteristic. However, as polythene does not stretch elastically it has a high level of hysteresis in a trial. Nonetheless, this does not limit granular jamming from being repeatable per se. Despite hysteresis or inelastic deformation shown in Figure 6, Figure 9, and Figure 11 , once air is reintroduced into the joint, it is effectively reset and ready to operate again. In other words, when the joint as a whole exhibits inelastic behavior or "permanent" deformation, may it be from the membrane or granules, the joint can retain its maximum stiffness characteristic upon unjamming and re-jamming the joint. The re-jamming process compensates for changes in previous granule packings and membrane deformations, as shown by the low variability in our results. This is unlike conventional materials where inelastic deformation is permanent and translates to poor repeatability. Thus, as a robotic mechanism, the variability between granular jamming trials is the important factor for its repeatability. For example, in the case of polythene in tension, while it exhibited an average of $42 \%$ hysteresis per trial, it only exhibited and average of $2.2 \%$ in variability between trials. These results provide design considerations for a variable stiffness flexible manipulator, where granular jamming is used to tune the rigidity of an endoscopic device, such as the device seen in Figure 1. The endoscope is soft to be dexterous and compliant when inserted, but can become rigid to provide the camera with a stable platform. Though previous works show that the granules impact the stiffness range and 
profile of the robotic device, our results show the membrane also plays a significant role $(\mathrm{p}<0.005$ between most membranes).

The conventional preconception is that a thin, flexible membrane is most ideal for granular jamming, such as latex rubber. However, our results show that this is not the case, with polythene consistently outperforming the rubber-based membranes in stiffness. At atmospheric pressure, the granule-filled joints for each of the membranes performed similarly to one another $(\mathrm{p}>0.1$ for nitrile and vitrile bending), with the exception of polythene. The rubber-like materials latex, nitrile, vinyl, and vitrile tended to have better hysteresis and low variability. However, when vacuumed to $55 \mathrm{kPa}$ and 10 $\mathrm{kPa}$, the joints began to behave much differently from each other. This could be attributed to the interaction between the granules and membrane. At atmospheric pressure, the granules play only a small role. When jammed, the membrane's ability to maintain stress on granules causes the joints to behave more solid-like. For example, during compression in atmosphere, the granules are pushed together and thusly expand the latex membrane. At $10 \mathrm{kPa}$ vacuum, the granules' attempt to expand the membrane is counteracted by the latex's constriction due to pressure. Thus, the measured stress increases 6-fold, as can be seen from Figure 11. On the other hand, nitrile only had a 3-fold increase. This may be from the less flexibility of nitrile, as the membrane-only tests in Figure 5 show that it experiences substantial hysteresis. Similarly for polythene, as the material's inherent properties resists expansion, the relative change between atmospheric and vacuum is only about a $50 \%$ increase in stiffness.

Additionally, the behaviors of the joints in the three overarching tests showed that, on a macro scale, the granular jamming system behaves as a different type of material in bending, tension, and compression, as the Young's modulus E change depending on the loading direction. Thus, future robotic manipulators using granular jamming must take the performance of desired tasks into consideration, as they differ.

From (34), changing the granule shape and surface friction can greatly increase the stiffness of the joint. Changing from smooth glass spheres to matte plastic cubes increased the stiffness by 1.8 times. From (32), stiffness can be increased by using smaller granules. Here, we show that the membrane properties are also a driving factor in achievable stiffness. Thus, future applications for granular jamming must use these factors in consideration.

\section{Conclusions}

This paper presents the characteristics of five clinically approved membrane materials for a granular jamming-based flexible robot. Latex and nitrile were found to be similar in bending and tension tests, and are "well-rounded" materials. In particular, latex not only has the least variance in $\mathrm{E}$ between the bending, tension, and compression tests, but also behaves distinctively between atmospheric, $55 \mathrm{kPa}$, and $10 \mathrm{kPa}$. Vinyl and vitrile tend to be quite varied between the load scenarios, working better than latex in some while worse in others. Polythene consistently achieved the highest E values for all the tests, which may be beneficial for applications focused on stiffness. However, for applications desiring a softer state or better stiffness range, latex may be better suited.

Future work includes further analysis of membrane to granule interactions and designing membranes to optimize such interactions. Such as in (32), a bumpy membrane, which increases the friction and couples the membrane to the granules, certain properties can be improved. The membrane coupling can improve the tensile performance of a granular jammed joint, as the jamming phenomenon begins to apply to the membrane, as well.

\section{Acknowledgements}

We would like to thank both the engineering and medical community for their helpful discussions and comments. The work described in this paper is partially supported by the Seventh Framework Programme of the European Commission in the framework of EU project STIFF-FLOP [grant agreement 287728] and the National Institute for Health Research (NIHR) Biomedical Research Centre based at Guy's and St Thomas' NHS Foundation Trust and King's College London. The views expressed are those of the authors and not necessarily those of the NHS, the NIHR or the Department of Health. 


\section{Bibliography}

1. Comparison of outcomes between pure laparoscopic vs robot-assisted laparoscopic radical prostatectomy: a study of comparative effectiveness based upon validated quality of life outcomes. Willis, Daniel L., et al., et al. 2011, BJU International, Vol. 109, pp. 898-905.

2. A Comparative Direct Cost Analysis of Pediatric Urologic Robot-Assisted Laparoscopic Surgery vs. Open Surgery: Could Robotic Surgery be Less Expensive? Rowe, Courtney K, et al., et al. 2011, Journal of Endourology.

3. Flexible Robotics. Vyas, L., et al., et al. 2, 2011, Bju International, Vol. 107, pp. 187-189.

4. Natural orifice translumenal endoscopic surgery (NOTES): a technical review. Auyang, Edward, et al., et al. s.l. : Springer New York, 2011, Surgical Endoscopy, Vol. 25, pp. 3135-3148.

5. Scopes Too Flexible... and Too Stiff. Loeve, A., Breedveld, P. and Dankelman, J. 3, 2010, Pulse, IEEE, Vol. 1, pp. 26-41.

6. SOFT ROBOTICS TECHNOLOGIES TO ADDRESS SHORTCOMINGS IN TODAY'S MINIMALLY INVASIVE SURGERY: THE STIFFFLOP APPROACH. Cianchetti, M., et al., et al. s.I. : Soft Robotics (SoRo), 2014.

7. Highly Articulated Robotic Probe for Minimally Invasive Surgery. Degani, A., et al., et al. 2008, 2008 30th Annual International Conference of the IEEE Engineering in Medicine and Biology Society, Vols 1-8, pp. 3273-3276.

8. Snake-like units using flexible backbones and actuation redundancy for enhanced miniaturization. Simaan, N. 2005, 2005 IEEE International Conference on Robotics and Automation (ICRA), Vols 1-4, pp. 3012-3017.

9. Mechanics Modeling of Tendon-Driven Continuum Manipulators. Camarillo, D. B., et al., et al. 6, 2008, leee Transactions on Robotics, Vol. 24, pp. 1262-1273.

10. A Novel Concept for Building a Hyper-Redundant Chain Robot. Ning, KeJun and Worgotter, F. 6, 2009, Robotics, IEEE Transactions on, Vol. 25, pp. 1237-1248.

11. An articulated universal joint based flexible access robot for minimally invasive surgery. Shang, J., et al., et al. 2011. pp. 1147-1152.

12. Three degrees-of-freedom joint for spatial hyper-redundant robots. Shammas, E., Wolf, A. and Choset, H. 2, s.I. : Elsevier, 2006, Mechanism and machine theory, Vol. 41, pp. 170-190.

13. Design and Analysis of a Soft Mobile Robot Composed of Multiple Thermally Activated Joints Driven by a Single Actuator. Cheng, Nadia, et al., et al. 2010, 2010 IEEE International Conference on Robotics and Automation (ICRA), pp. 5207-5212.

14. Design and Control of Concentric-Tube Robots. Dupont, P.E., et al., et al. 2, 2010, Robotics, IEEE Transactions on, Vol. 26, pp. 209-225.

15. Toward a hybrid snake robot for single-port surgery. Mahvash, M. and Zenati, M. 2011. pp. 5372-5375.

16. Nonlinear dynamics: Jamming is not just cool any more. Liu, Andrea J. and Nagel, Sidney R. 1998, Nature, Vol. 296, pp. 21-22.

17. Universal robotic gripper based on the jamming of granular material. Brown, E., et al., et al. 44, 2010, Proceedings of the National Academy of Sciences of the United States of America, Vol. 107, pp. 18809-18814. 
18. Design and Analysis of a Robust, Low-cost, Highly Articulated manipulator enabled by jamming of granular media. Cheng, Nadia G., et al., et al. 2012, 2012 IEEE International Conference on Robotics and Automation (ICRA), pp. 43284333.

19. JSEL: Jamming Skin Enabled Locomotion. Steltz, E., et al., et al. 2009, 2009 IEEE/RSJ International Conference on Intelligent Robots and Systems, (IROS)., pp. 5672-5677.

20. Jamming as an enabling technology for soft robotics. Steltz, E., et al., et al. 2010, Society of Photo-Optical Instrumentation Engineers (SPIE) Conference Series, Vol. 7642, p. 63.

21. Wearable haptic display by the use of a particle mechanical constraint. Mitsuda, T., et al., et al. 2002, Proceedings. 10th Symposium on HAPTICS 2002, pp. 153-158.

22. Vacuum packed particles as flexible edoscope guides with controllable rigidity. Loeve, Arjo J., et al., et al. 2010, Granular Matter, Vol. 12, pp. 543-554.

23. STIFF-FLOP Surgical Manipulator: mechanical design and experimental characterization of the single module.

Cianchetti, M., et al., et al. Tokyo : IEEE/RSJ International Conference on Intelligent Robots and Systems (IROS), 2013.

24. A modular soft manipulator with variable stiffness. Ranzani, T., et al., et al. 2013.

25. The vacuum splint: an aid in emergency splinting of fractures. Letts, RM and Hobson, DA. 7, s.I. : Canadian Medical Association, 1973, Canadian Medical Association Journal, Vol. 109, p. 599.

26. Fundamental problems in statistical physics of jammed packings. Edwards, SF, Grinev, DV and Brujic, J. 1-2, 2003, Physica a-Statistical Mechanics and Its Applications, Vol. 330, pp. 61-76.

27. Statistical mechanics of granular materials: stress propagation and distribution of contact forces. Edwards, SF and Grinev, DV. 4, 2003, Granular Matter, Vol. 4, pp. 147-153.

28. Jamming phase diagram for frictional particles. Ciamarra, M.P., et al., et al. 2009, Arxiv preprint arXiv:0912.3140.

29. Granular packing: numerical simulation and the characterisation of the effect of particle shape. Guises, Romain, et al., et al. 2009, Granular Matter, Vol. 11, pp. 281-292.

30. Computer Simulation of Granular Materials. Saadatfar, M. 1, 2009, Computing in Science Engineering, Vol. 11, pp. 66-74.

31. Adapting granular materials through artificial evolution. Miskin, Marc Z and Jaeger, Heinrich M. s.I. : Nature Publishing Group, 2013, Nature materials.

32. Design of a variable stiffness flexible manipulator with composite granular jamming and membrane coupling. Jiang, Allen, et al., et al. 2012. pp. 2922-2927.

33. The Core-Snake, the Variable Stiffness Laparoscopic Camera. Jiang, Allen, et al., et al. 2013.

34. A Variable Stiffness Joint by Granular Jamming. Jiang, Allen, et al., et al. 2012.

35. The Mullins effect in uniaxial extension and its influence on the transverse vibration of a rubber string. Johnson, MA and Beatty, MF. 2, s.I. : Springer, 1993, Continuum Mechanics and Thermodynamics, Vol. 5, pp. 83-115. 
Table 1: Membrane-only tensile test

\begin{tabular}{|l|c|c|c|c|}
\hline Membrane Type & Stress (MPa) & Hysteresis & Variability (MPa) & E (MPa) \\
\hline Latex & 0.01 & 0.07 & 0.006 & 0.06 \\
\hline Nitrile & 0.01 & 0.52 & 0.004 & 0.06 \\
\hline Vinyl & 0.01 & 0.24 & 0.006 & 0.06 \\
\hline Vitrile & 0.02 & 0.20 & 0.007 & 0.08 \\
\hline Polythene & 0.17 & 0.53 & 0.060 & 0.83 \\
\hline
\end{tabular}

Table 2: Bending test

\begin{tabular}{|l|c|c|c|c|c|}
\hline Membrane Type & Pressure (kPa) & Force (N) & Hysteresis & Variability (N) & E (MPa) \\
\hline Latex & 101 & 0.36 & 0.42 & 0.01 & 0.31 \\
& 55 & 1.72 & 0.43 & 0.08 & 1.47 \\
& 10 & 2.03 & 0.51 & 0.17 & 1.74 \\
\hline Nitrile & 101 & 0.39 & 0.36 & 0.02 & 0.33 \\
& 55 & 1.86 & 0.40 & 0.09 & 1.60 \\
& 10 & 2.12 & 0.51 & 0.14 & 0.11 \\
\hline Vinyl & 101 & 0.13 & 0.50 & 0.12 & 1.22 \\
& 55 & 1.42 & 0.44 & 0.21 & 0.32 \\
\hline Vitrile & 10 & 0.27 & 0.55 & 0.02 & 1.00 \\
& 101 & 1.16 & 0.69 & 0.11 & 1.52 \\
\hline Polythene & 55 & 1.77 & 0.49 & 0.18 & 0.41 \\
& 10 & 0.48 & 0.48 & 0.13 & 2.08 \\
\hline
\end{tabular}

Table 3: Tensile test

\begin{tabular}{|l|c|c|c|c|c|}
\hline Membrane Type & Pressure (kPa) & Stress (MPa) & Hysteresis & Variability (MPa) & E (MPa) \\
\hline Latex & 101 & 0.01 & 0.40 & 0.0002 & 0.06 \\
& 55 & 0.03 & 0.36 & 0.0010 & 0.12 \\
& 10 & 0.04 & 0.30 & 0.0011 & 0.20 \\
\hline Nitrile & 101 & 0.02 & 0.26 & 0.0009 & 0.06 \\
& 55 & 0.04 & 0.28 & 0.0020 & 0.19 \\
& 10 & 0.05 & 0.30 & 0.0005 & 0.19 \\
\hline Vinyl & 101 & 0.02 & 0.37 & 0.0006 & 0.08 \\
& 55 & 0.02 & 0.35 & 0.0009 & 0.09 \\
\hline Vitrile & 10 & 0.02 & 0.43 & 0.0012 & 0.08 \\
& 101 & 0.06 & 0.30 & 0.0006 & 0.25 \\
& 55 & 0.08 & 0.27 & 0.0013 & 0.33 \\
\hline Polythene & 10 & 0.11 & 0.40 & 0.0010 & 0.43 \\
& 101 & 0.14 & 0.42 & 0.0030 & 0.53 \\
\hline
\end{tabular}

Table 4: Compression test

\begin{tabular}{|l|c|c|c|c|c|}
\hline Membrane Type & Pressure (kPa) & Stress (MPa) & Hysteresis & Variability (MPa) & E (MPa) \\
\hline Latex & 101 & 0.01 & 0.12 & 0.0002 & 0.11 \\
& 55 & 0.03 & 0.06 & 0.0009 & 1.06 \\
& 10 & 0.06 & 0.04 & 0.0006 & 2.66 \\
\hline
\end{tabular}




\begin{tabular}{|l|c|c|c|c|c|}
\hline Nitrile & 101 & 0.01 & 0.28 & 0.0003 & 0.14 \\
& 55 & 0.02 & 0.02 & 0.0002 & 0.86 \\
\hline Vinyl & 10 & 0.03 & 0.09 & 0.0010 & 1.37 \\
& 101 & 0.01 & 0.30 & 0.0006 & 0.17 \\
& 55 & 0.03 & 0.07 & 0.0007 & 2.13 \\
\hline Vitrile & 10 & 0.08 & 0.05 & 0.0016 & 0.30 \\
& 101 & 0.01 & 0.09 & 0.0005 & 0.91 \\
\hline Polythene & 55 & 0.02 & 0.21 & 0.0005 & 1.26 \\
& 10 & 0.05 & 0.20 & 0.0012 & 2.25 \\
& 55 & 0.09 & 0.09 & 0.0014 & 3.31 \\
\hline
\end{tabular}

